\title{
La elección de la metodología de aprendizaje como fuente de innovación en los estudios de administración de empresas de la Universidad de Andorra
}

\author{
Rosa Maria Mariño-Mesías a, Josep Fortó-Areny ${ }^{\text {b }}$, Betlem Sabrià-Bernadóc \\ a'Universitat d’Andorra rmarino@uda.ad, bUniversitat d’Andorra jforto@uda.ad, 'Universitat \\ d’Andorrabsabria@uda.ad.
}

\begin{abstract}
At the time of designing a new curriculum of business administration studies of the University of Andorra (UdA), adapted to the European Higher Education Area (EHEA) and taking into account the high percentage of students who combine their studies with professional activities, several teaching innovations were introduced in order to improve the accessibility in these studies.

This paper presents the implemented learning methodology that allows students to choose their own mode of study (face-to-face, online or blended) according to their preferences. Students, not only has a total flexibility to choose the type of learning modality, but they also have the possibility of changing it throughout their training. A literature review aimed to elaborate a questionnaire that has allowed to know the opinion of the teaching staff about this new methodology.

The main conclusion of the study shows that aspects related to flexibility, access to information and support for learning are the best valued. In this line, the UdA also improves the accessibility and participatory equity promoted by the EHEA.
\end{abstract}

Keywords: Innovation, methodology, personalization, flexibility, teaching staff, virtual learning environment, bachelor of business administration, University of Andorra.

\begin{abstract}
Resumen
En el momento de diseñar un nuevo plan de estudios de administración de empresas de la Universitat d'Andorra (UdA), adaptado al Espacio Europeo de Educación Superior (EEES) y teniendo en cuenta el elevado porcentaje de estudiantes de la UdA que compaginan sus estudios con actividades profesionales, se introdujeron innovaciones docentes para mejorar la accesibilidad en estos estudios.

En este trabajo se presenta la metodología de aprendizaje implantada que permite a los estudiantes elegir su propia modalidad de estudio (presencial, virtual o semipresencial) en función de sus preferencias. Los estudiantes, no solo tienen una flexibilidad total para elegir el tipo de modalidad de aprendizaje, sino que también tienen la posibilidad de cambiarla a lo largo de su formación.

A partir de la revisión de la literatura se ha elaborado un cuestionario que ha permitido conocer la opinión del profesorado sobre esta nueva metodología.

La conclusión principal del estudio muestra que los aspectos relacionados con la flexibilidad, el acceso a la información y el soporte al aprendizaje son los mejor valorados. En esta línea, la UdA también mejora la accesibilidad y la equidad participativa promovida por el EEES.
\end{abstract}

Palabras clave: Innovación, metodología, personalización, flexibilidad, profesorado, entorno virtual de aprendizaje, bachelor en administración de empresas, Universidad de Andorra. 
La elección de la metodología de aprendizaje como fuente de innovación en los estudios de administración de empresas de la Universidad de Andorra

\section{Introducción}

El Principado de Andorra es un microestado europeo situado en la zona pirenaica con una extensión aproximada de $468 \mathrm{~km}^{2}$ y una población de 74.794 habitantes (Govern d'Andorra, Departament d'Estadística, 2018).

Existe un buen vínculo entre el Principado de Andorra y sus estados vecinos, España y Francia, que hacen que la influencia de estos países sea grande en muchos ámbitos y, en concreto, en el de la formación. En el país conviven tres sistemas que configuran su red educativa pública -el andorrano, el español y el francés-. Cada uno de estos sistemas sigue los planes de estudios y los programas que emanan de los Ministerios de Educación de los países respectivos.

La sociedad andorrana está experimentando grandes transformaciones que afectan a la manera de vivir de todos los ciudadanos, y en especial, la de los jóvenes. En el Principado de Andorra, el nivel formativo de los jóvenes en relación al de las generaciones anteriores se ha incrementado. El mercado laboral andorrano se caracteriza por tener una gran rotación y segmentación, en el que los jóvenes se encuentran en situaciones de mayor precariedad laboral (CRES, 2007).

A pesar de los esfuerzos llevados a cabo en el Principado de Andorra durante las últimas décadas en el ámbito de la formación y la educación, entre los que destaca la creación de la Universidad de Andorra, el nivel formativo de la población se ve condicionado de manera muy notable por la importancia de los flujos migratorios. Estas migraciones, básicamente representan una mano de obra no cualificada y, en consecuencia, unos déficits formativos elevados en el lugar de trabajo (Forma, 2007).

En el año 1988 se inició la educación superior en el Principado de Andorra. La Universidad de Andorra (UdA) es la única universidad pública del país. Durante el curso académico 2016-2017, se matricularon 1.243 estudiantes y dispone de un personal fijo formado por 34 personas, 22 de las cuales forman parte del personal docente e investigador. El resto de profesorado -eventual, colaborador y visitante- supone un total de 288 personas que provienen de otras universidades y organizaciones profesionales, tanto nacionales como extranjeras.

La estructura de la UdA es pequeña y flexible. Estos factores influyen positivamente en la adaptación a los cambios constantes que requiere la sociedad del conocimiento, pero también conllevan una serie de dificultades añadidas, ya que hay que coordinar colectivos e instituciones colaboradoras muy diversas.

A lo largo de los años, la UdA ha ido transformando sus prácticas educativas y el curso 2010-2011, cuando adaptó sus titulaciones al Espacio Europeo de Educación Superior (EEES), decidió ofrecer algunas de sus titulaciones siguiendo los tres modelos de docencia: presencial, semipresencial y virtual.

\section{Objetivos}

Tal como pone de manifiesto la revisión de la literatura, la demanda de educación virtual en la educación superior está aumentando. También aumenta la competencia entre universidades y, en consecuencia, invierten recursos significativos para mejorar la calidad de sus ofertas (Martínez-Caro, Cegarra-Navarro y Cepeda-Carrión, 2015). En este sentido, se considera que la apuesta por el uso de la tecnología puede mejorar la calidad de la educación (Mayisela, 2014; Nicolaou, Nicolaidou y Constantinou, 2005). 
Los estándares y directrices para el aseguramiento de la calidad en el EEES (ESG, 2015) destacan la importancia del aprendizaje, enseñanza y evaluación centrados en el estudiante, ya que de esta forma se motiva y promueve su participación en el proceso de aprendizaje. Tal como se especifica en la directriz que corresponde a este estándar, es necesario que las universidades presten atención al diseño y a la modalidad de enseñanza de los programas de estudio, así como a la evaluación de los resultados. En esta misma línea, a la hora de asignar, planificar y ofrecer los recursos de aprendizaje y de soporte a los estudiantes, se tienen en cuenta las necesidades de una población estudiantil diversa y las modalidades flexibles de aprendizaje y de enseñanza.

Según el trabajo desarrollado por Martínez-Caro et al. (2015), la mayoría de los investigadores coinciden en que los principales factores que afectan la satisfacción del estudiante son la interacción entre el profesorado y el estudiante, la interacción entre estudiantes, los contenidos del aprendizaje y, la flexibilidad y apoyo del sistema. Con la introducción de las nuevas tecnologías de la información y la comunicación (TIC), el número de herramientas para promover la interacción entre los participantes de una formación en línea ha aumentado enormemente.

Algunos autores (Castaño, Jenaro, Flores, 2017; López-Pérez, Pérez-López, RodríguezAriza, 2011), sostienen que las TIC proporcionan a los estudiantes y al profesorado un entorno de aprendizaje innovador para estimular y mejorar el proceso de enseñanza y aprendizaje y, en este contexto, se están desarrollando nuevos conceptos educativos, como el aprendizaje combinado o semipresencial. Esta innovación también ha supuesto un cambio de paradigma en cuanto a recursos e infraestructuras, tanto en el modelo de gestión, como en el de organización académica.

Asimismo, según Alebaikan y Troudi (2010) el aprendizaje combinado ha demostrado un considerable efecto positivo en el proceso de aprendizaje y proporciona flexibilidad y accesibilidad al aprendizaje tradicional, ya que integra el aprendizaje cara a cara con el aprendizaje a distancia. Según estos mismos autores, algunas universidades promueven el aprendizaje combinado como una estrategia, ofreciendo flexibilidad en el momento y el lugar de aprendizaje, y los estudiantes prefieren el aprendizaje combinado sobre el aprendizaje en línea.

A partir de la implementación de la innovación docente en el bachelor en administración de empresas (BAE) de la UdA, que combina los tres modelos de docencia: presencial, semipresencial y virtual, permitiendo una flexibilidad total al estudiante a la hora de escoger su modalidad de aprendizaje, el primero de los objetivos de este trabajo es presentar el perfil de los estudiantes de nuevo acceso a la UdA que han seguido estas metodologías.

Cabe destacar que en otro de los ESG desarrollados en el EEES para el aseguramiento de la calidad, se señala la importancia del papel del profesorado para crear una experiencia de calidad en los estudiantes y para facilitar la adquisición de conocimientos, competencias y habilidades. Tal como también se describe en la directriz correspondiente del estándar, las instituciones de educación superior son las principales responsables de la calidad de sus trabajadores y de proporcionarles un entorno que les permita llevar a cabo sus tareas con eficacia. Una de las características que debe presentar este entorno es fomentar la innovación en los métodos de enseñanza y el uso de las nuevas tecnologías.

Aunque el aprendizaje combinado requiere un alto nivel de disciplina por parte de los estudiantes, en la literatura existe consenso en que uno de los elementos fundamentales para el éxito de estas metodologías es el profesorado (Alebaikan y Troudi, 2010; Castaño et al., 2017; García-Varcálcel, Hernández-Martín y Recamán-Payo, 2012; Mayisela, 2014). En particular, su nivel de habilidad técnica y su desarrollo profesional en este tipo de docencia 
en línea (Alebaikan y Troudi, 2010; Tyilo, 2017). Asimismo, otro de los elementos que se consideran necesarios es contar con unas infraestructuras adecuadas para desarrollar este tipo de enseñanza (Nicolaou et al., 2005).

Siguiendo el hilo de la importancia del papel que desempeña el personal docente en el éxito de estas metodologías, el segundo de los objetivos de este trabajo es presentar el perfil del profesorado del BAE y conocer su opinión o valoración sobre esta metodología docente en línea, tal como también han hecho otros trabajos (Ferro, Martínez y Otero, 2009; Mayisela, 2014). Los trabajos de Lai, Lam y Lim (2016) y de Nicolaou et al. (2005), además de la opinión del profesorado, también analizan la opinión de los estudiantes. Otros autores, como por ejemplo, Martínez-Caro et al. (2015), McGorry (2003) y Wang (2003), en cambio, se han centrado únicamente en el punto de vista de los estudiantes, para proponer modelos de evaluación de la calidad en las formaciones virtuales o para evaluar su satisfacción en este tipo de formaciones.

Tal como se ha especificado a lo largo de este apartado del trabajo, los dos objetivos de esta investigación son los siguientes:

Por un lado, se pretende mostrar el perfil de los estudiantes del BAE que han seguido los tres modelos de docencia: presencial, semipresencial y virtual. Y por otro, presentar las características del profesorado de la titulación y, además, conocer su opinión sobre el uso del campus virtual como soporte a la flexibilidad en la elección de la metodología.

\section{Desarrollo de la innovación}

La UdA dispone de un entorno virtual de aprendizaje (EVA) propio desde el curso 20042005. Este entorno estaba integrado con tecnología Lotus Notes y tenía los dos objetivos siguientes a corto plazo:

- Ofrecer un valor añadido a los programas de formación presenciales existentes, basados en grupos reducidos de estudiants y en una atención personalizada.

- Permitir el desarrollo de nuevos programas de formación de contenidos específicos andorranos, siguiendo una metodología virtual.

En el 2009, la UdA cambió su gestor de cursos virtuales hacia la plataforma Moodle, que se fundamenta en una óptica de construcción del conocimiento basado en el diálogo entre los participantes. Después de un semestre de pruebas, en el curso 2010-2011 se instauró en todos los estudios de la UdA.

Para Zúñiga y Arnáez (2010) los entornos virtuales son comunidades virtuales de aprendizaje que, por medio de las TIC, brindan a los docentes y estudiantes espacios de aprendizaje activo donde la reflexión, la discusión y el intercambio de información producen experiencias significativas y construcción de conocimientos dentro de un contexto social virtual, siendo la capacidad de autoformación, una actividad imprescindible. Además, tal como sostiene Martínez-Caro (2011), estos entornos se centran en el estudiante y facilitan la flexibilidad del aprendizaje interactivo (en cualquier momento y lugar).

El modelo pedagógico subyacente en el nuevo campus virtual de la UdA está basado en el sociconstructivismo de Vigotsky (1984). En este modelo, el estudiante se considera constructor activo de su propio aprendizaje, a través de las interacciones con los demás. Pasa de ser un receptor pasivo de contenidos estáticos y memorísticos a ser el principal protagonista y a resolver problemas utilizando los conocimientos adquiridos. 
En función del grado de presencialidad o virtualidad entre el profesorado y el alumnado, se diferencian los tres tipos de modelos de docencia siguientes (Area y Adell, 2009; Area, San Nicolás y Fariña, 2010):

- La docencia presencial, en la que el aula virtual es un recurso complementario. En este caso, lo relevante sigue siendo el proceso de enseñanza presencial del aula y el profesor sigue haciendo lo de siempre, con la ayuda de las TIC.

- La docencia semipresencial, docencia mixta o blended learning, en la que se yuxtaponen los procesos de enseñanza-aprendizaje presenciales con otros que se desarrollan a distancia, mediante el uso de dispositivos electrónicos. Para Torrisi-Steele y Drew (2013), el blended learning se refiere al uso de la tecnología con la enseñanza presencial. En este caso, el aula virtual es un recurso de apoyo a la enseñanza presencial y constituye un espacio en el que el profesor genera y desarrolla acciones diversas para que sus alumnos aprendan. Asimismo, debe elaborar materiales y actividades para que el estudiante las desarrolle autónomamente fuera del contexto de la clase tradicional.

- La docencia virtual o e-learning, en la que el aula virtual es el único espacio educativo. En este caso, el material o recursos didácticos multimedia cobran una especial relevancia. La interacción comunicativa dentro del aula virtual se convierte en un factor clave y sustantivo para el éxito de la metodología.

La enseñanza y el aprendizaje usando las TIC ha supuesto un cambio de mentalidad para el docente y para el estudiante. Según Alabart, Escofet y Vilá (2008) este cambio de mentalidad es complejo pero es valorado como una transición obligatoria y, al mismo tiempo, irreversible en el contexto universitario. La dificultad más notoria que tienen las TIC para convertirse en herramientas que puedan generar conocimiento reside en encontrar, concebir, desarrollar e implementar usos que lleguen a ser pedagógicamente significativos y que favorezcan la apropiación socioeducativa de los recursos aportados a los estudiantes (Levis, 2011).

El EEES es un marco educativo ambicioso y complejo que la mayoría de países europeos, incluyendo el Principado de Andorra, han integrado con el objetivo de favorecer la convergencia europea, en materia de educación. Este nuevo escenario supone una renovación de los métodos docentes, con la aplicación de un nuevo modelo de enseñanza y de aprendizaje. Se trata de conseguir unos objetivos de formación que contemplen tanto la adquisición de conocimientos, como la adquisición de las competencias académicas y profesionales. En este sistema se reducen las horas de docencia directa o clases magistrales y se da más importancia a todo el proceso de aprendizaje del estudiante, ya que tiene un rol más activo y participativo durante todo su proceso de formación. En este escenario, las TIC son imprescindibles para gestionar y cuantificar de una manera eficiente el trabajo autónomo de los estudiantes y el EVA permite hacer un seguimiento del aprendizaje no presencial de los estudiantes.

Con la Ley 12/2008, del 12 de junio, de ordenación de la educación superior (Butlletí Oficial del Principat d'Andorra -BOPA-, 2008), el Principado de Andorra adecua su ordenamiento jurídico en materia de educación superior, de acuerdo con los principios del EEES. Para dar respuesta a la necesidad de formar profesionales con unos conocimientos básicos en el campo de la administración de empresas, se creó el título estatal del Bàtxelor en Administració d'Empreses, el cual proporciona una formación teórica y práctica, multidisciplinar y generalista, en consonancia con las características del mercado laboral andorrano (BOPA, 2010). La obtención de este título garantiza los conocimientos y las competencias necesarias para desarrollar las tareas principales vinculadas a la gestión 
La elección de la metodología de aprendizaje como fuente de innovación en los estudios de administración de empresas de la Universidad de Andorra

empresarial, principalmente en los ámbitos contable y financiero, comercial, tributario e internacional.

Esta formación de 180 créditos europeos (BOPA, 2011) se caracteriza por tener una metodología de aprendizaje flexible, que permite al estudiante disponer de un amplio abanico de posibilidades a la hora de decidir la carga lectiva que quiere asumir cada semestre. La totalidad de créditos europeos del plan de estudios se desglosa en 150 créditos obligatorios, 18 créditos optativos y 12 créditos de libre elección, los cuales se distribuyen en seis semestres, con una carga de 30 créditos cada uno.

El plan de estudios se apoya en bloques formativos relativos a la formación específica, que facilita una formación técnica, una formación en TIC, una formación instrumental y una formación en idiomas.

Para responder al primero de los objetivos de este trabajo, por un lado, se han extraído los principales datos sobre las características de los estudiantes, a partir de la encuesta que responden los nuevos estudiantes de la UdA sobre cuestiones generales de su entorno, al inicio de cada curso universitario, desde el curso 2012-2013. Los estudiantes que responden el cuestionario sobre su entorno son los que siguen la modalidad presencial o semipresencial, puesto que este cuestionario se distribuye y se responde, físicamente en la universidad. A la hora de formalizar la matrícula, una de las cuestiones que se les pregunta a los estudiantes del BAE, es que indiquen la modalidad de estudios que van a seguir (en función de sus preferencias), aunque esta modalidad puede variar a lo largo del curso académico en cualquiera de las asignaturas de la titulación, puesto que el sistema de evaluación es el mismo para cualquiera de las tres modalidades.

Con el propósito de responder al segundo de los objetivos de este estudio, se ha elaborado un cuestionario dirigido a la totalidad del profesorado del BAE. Concretamente, a partir de la revisión de la literatura se ha adaptado el cuestionario utilizado en la investigación de Ferro et al. (2009) sobre las ventajas de las TIC, desde el punto de vista de los docentes universitarios españoles. Este cuestionario ha permitido recoger la opinión del profesorado del BAE y extraer las principales conclusiones relativas a los aspectos más destacados de la metodología de docencia virtual utilizada.

El profesorado del BAE está formado por un total de 22 personas a las que se les envió un cuestionario (ver tabla 1), en el que se les pedía que valorasen en una escala de respuesta Likert de 5 opciones (en la que 1 significaba que ese ítem no tenía ninguna importancia y 5 significaba que su importancia era máxima), los siguientes ítems sobre la importancia del uso del campus virtual del BAE como herramienta de flexibilización de las metodologías de aprendizaje. La recogida de datos se realizó durante el mes de junio de 2017 y la tasa de respuesta fue del $100 \%$.

Tabla 1. Cuestionario destinado al profesorado del BAE

\begin{tabular}{|l|}
\hline \multicolumn{1}{|c|}{ Preguntas } \\
\hline P1: Mejora la comunicación entre los estudiantes \\
\hline P2: Permite una enseñanza más personalizada \\
\hline P3: Mejora la eficacia educativa \\
\hline P4: Facilita que los procesos formativos sean más abiertos y flexibles \\
\hline P5: Aumenta el interés y la motivación de los estudiantes \\
\hline P6: Mejora la comunicación profesor-estudiante \\
\hline P7: Da acceso rápido a la información \\
\hline
\end{tabular}




\begin{tabular}{|l|}
\hline \multicolumn{1}{|c|}{ Preguntas } \\
\hline P8: Permite que el profesorado tenga más tiempo para otras tareas \\
\hline P9: Facilita la interacción con la información \\
\hline $\begin{array}{l}\text { P10: Facilita la realización de actividades complementarias de apoyo } \\
\text { al aprendizaje }\end{array}$ \\
\hline P11: Rompe las barreras espacio-tiempo \\
\hline P12: Facilita el seguimiento de las asignaturas \\
\hline
\end{tabular}

Fuente: Ferro et al. (2009)

Con el objetivo de analizar los datos, se han utilizado técnicas de estadística descriptiva y técnicas de estadística inferencial, a partir del software estadístico R.

Dado que las variables no siguen una distribución Normal, se ha utilizado la prueba no paramétrica U de Mann-Whitney-Wilcoxon.

\section{Resultados}

En primer lugar, se presentan los resultados en relación al primero de los objetivos de este trabajo, es decir, en relación al perfil de los estudiantes del BAE.

Estos primeros resultados sobre el perfil de los estudiantes se han obtenido a partir del proceso de matrícula de la totalidad de los estudiantes matriculados en el BAE, desde el curso 2012-2013.

La media de edad de la primera matrícula realizada a los estudios de BAE es de 28,2 años, con una desviación típica de 7,9. Las edades oscilan entre los 18 y los 54 años y el $60 \%$ de los estudiantes tiene una edad superior a los 21 años.

Teniendo en cuenta que el BAE tiene una duración de tres años, hemos distribuido la edad en dos categorías: edad inferior o igual a 21 años y edad superior a 21 años, y hemos relacionado esta variable con la modalidad de matrícula.

El 66\% de los estudiantes presenciales tiene una edad inferior o igual a los 21 años, mientras que el 89\% de los estudiantes virtuales tiene una edad superior a los 21 años. Por último, el $64 \%$ de los estudiantes que siguen la modalidad semipresencial son mayores de 21 años.

A diferencia de los resultados obtenidos en el estudio Via Universitària (Sintes, 2016), realizado por la Xarxa Vives d'Universitats, que engloba 19 universidades de España, Francia y el Principado de Andorra en el que el 85,8\% de los estudiantes son presenciales, en el BAE, solo un $45,8 \%$ de los estudiantes optan por la modalidad presencial y asisten a clase regularmente. El $41,2 \%$ que siguen la modalidad virtual asisten a clase de forma muy esporádica y un $13 \%$ siguen la modalidad semipresencial.

Tal como se muestra en la tabla 2, la prueba de independencia Chi-cuadrado nos confirma que la edad es un factor determinante en la elección de la modalidad de matrícula a un nivel de significación del 99,99\% (p_value <0,000).

Tabla 2. Prueba Chi-cuadrado Edad-Modalidad de matrícula

\begin{tabular}{|l|c|c|c|}
\hline & Valor & gl & $\begin{array}{c}\text { Sig. asintótica } \\
\text { (bilateral) }\end{array}$ \\
\hline Chi-cuadrado de Pearson & $167,082(\mathrm{a})$ & 66 & $\mathbf{0 0 0}$ \\
\hline
\end{tabular}

(cc) B EY-NC-ND 2018, Universitat Politècnica de València

Congreso In-Red (2018) 
La elección de la metodología de aprendizaje como fuente de innovación en los estudios de administración de empresas de la Universidad de Andorra

\begin{tabular}{|l|c|c|c|}
\hline Razón de verosimilitudes & 197,637 & 66 &, $\mathbf{0 0 0}$ \\
\hline N de casos válidos & 322 & & \\
\hline
\end{tabular}

Siguiendo con el perfil de los estudiantes, a continuación se muestran los resultados que se han obtenido a partir de los datos de la encuesta sobre el entorno de los estudiantes. Tal como ya se ha especificado anteriormente, este cuestionario está dirigido a los estudiantes que siguen la modalidad presencial o semipresencial.

En la figura 1 se presenta la distribución de los estudiantes en función de la vía de acceso. Se constata que la mayoría de los estudiantes (presenciales o semipresenciales), el 48,65\%, proviene de un bachillerato general, pero también muchos de ellos (el 22,52\%) proceden de programas de formación profesional superior: Diploma profesional avanzado (DPA) -en el Principado de Andorra- o Ciclo Formativo de Grado Superior (CFGS). La vía de acceso del $21,62 \%$ de los estudiantes es a partir de un bachillerato profesional y el resto de los estudiantes, provienen de otras vías.

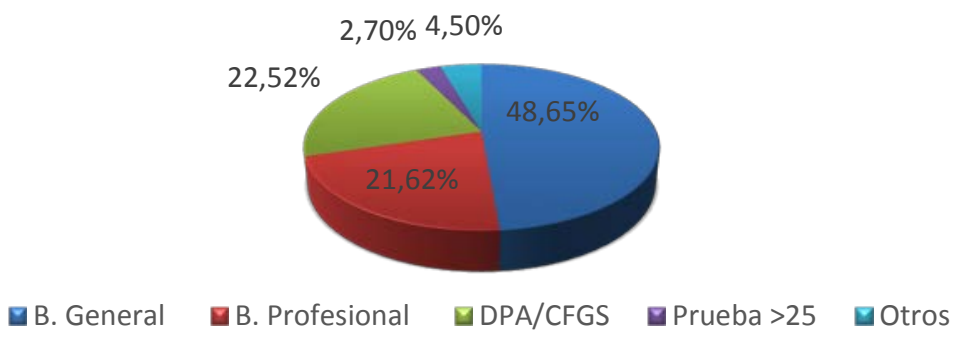

Fig. 1 Distribución de los estudiantes en función de la vía de acceso

Como ya hemos apuntado anteriormente, una característica importante de los estudiantes presenciales o semipresenciales del BAE es que muchos de ellos trabajan a jornada completa, media jornada o bien por horas. Tal como se muestra en la figura 2, tan solo un $45,95 \%$ de los estudiantes tienen dedicación exclusiva a los estudios. Se observa que más del 20\% trabaja más de 20 horas por semana, un 21,62\%, entre 10 y 20 horas, y un 11,71\% dedica menos de 10 horas al ámbito profesional.

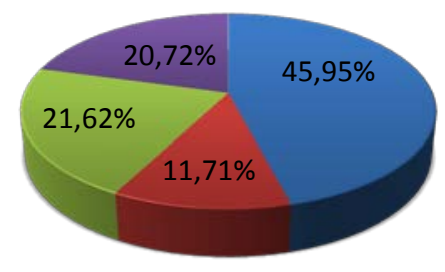

घ0h $\mathbf{a}$ Menos de $10 \mathrm{~h}$ Entre 10 y $20 \mathrm{~h}$ Más de $20 \mathrm{~h}$

Fig. 2 Distribución de los estudiantes en función de las horas de trabajo semanal remunerado

(cc) EY-NC-ND 2018, Universitat Politècnica de València

Congreso IN-RED (2018) 
A continuación, y en relación al segundo de los objetivos de este trabajo, se describe el perfil del profesorado del BAE y se muestran los resultados obtenidos a partir del cuestionario distribuido entre el profesorado.

El profesorado de la UdA está formado por personal fijo y mayoritariamente, por personal colaborador. Este último proviene, tanto de empresas del país, como de universidades de fuera. La combinación de docentes fijos y colaboradores externos es una característica diferencial de la UdA, que garantiza, tanto la solidez de la institución, como el dinamismo en la transmisión de conocimientos y el alcance internacional de su oferta formativa.

En la titulación concreta del BAE, aproximadamente el $60 \%$ del profesorado es fijo y el $40 \%$ restante, colaborador. Clasificando el profesorado por género, se observa que se está cerca de la paridad, ya que un $55 \%$ son hombres y un $45 \%$ mujeres.

La edad media del profesorado es de 43,5 años, la mediana de la serie de edades es de 45,5 años y la desviación típica es de 12,6 años. Esto indica que existe una importante dispersión de edades en el conjunto del profesorado. En la figura 3 se muestra el reparto del profesorado en función de la edad.

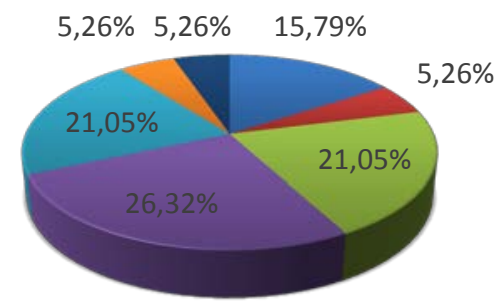

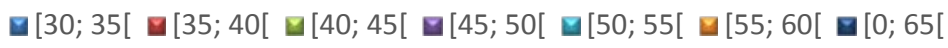

Fig. 3 Distribución del profesorado según su edad

A continuación (ver figura 4), se muestra la tipología del profesorado en función de los años de experiencia docente. Aproximadamente un $70 \%$ del profesorado tiene más de 10 años de experiencia. La experiencia media es de 17,3 años, la desviación típica es de 11,4 años y la mediana de la serie es de 22 años.
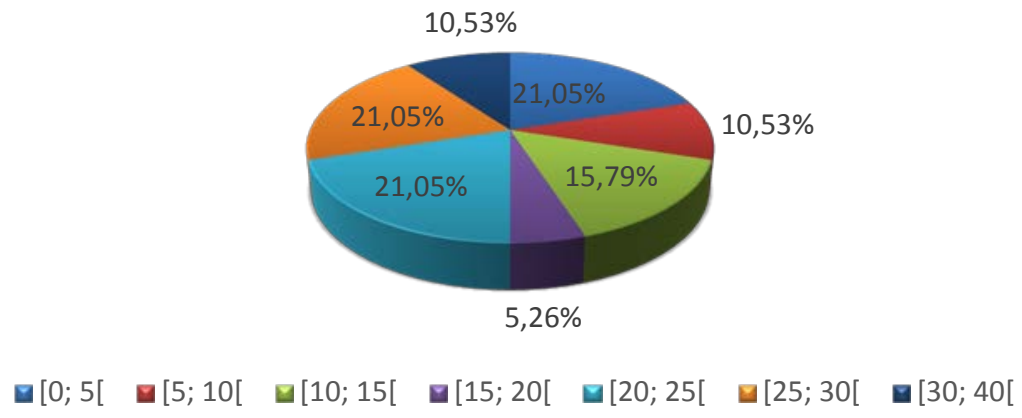

Fig. 4 Distribución del profesorado en función de los años de experiencia docente

En cuanto al análisis de los ítems que respondió el profesorado (ver figura 5), el mejor valorado fue el ítem que considera que el uso del campus virtual da acceso rápido a la información, con un valor medio de 4,59, seguido del ítem rompe las barreras espacio- 
La elección de la metodología de aprendizaje como fuente de innovación en los estudios de administración de empresas de la Universidad de Andorra

tiempo, con una puntuación igual a 4,45. Estos dos ítems también han sido los que han obtenido una desviación típica inferior $(0,67)$.

Contrariamente, el ítem con el valor más bajo fue el que considera que el uso del campus virtual permite que el profesorado tenga más tiempo para otras tareas, con un valor de 2,27, seguido del ítem aumenta el interés y la motivación de los estudiantes, con un valor medio de 2,64. Los valores de la desviación típica para estos dos ítems fueron de 1,16 y de 1 , respectivamente.

La mayor dispersión de opiniones en los encuestados se ha dado en el ítem mejora la comunicación entre los estudiantes, con un valor de desviación típica igual a 1,41.

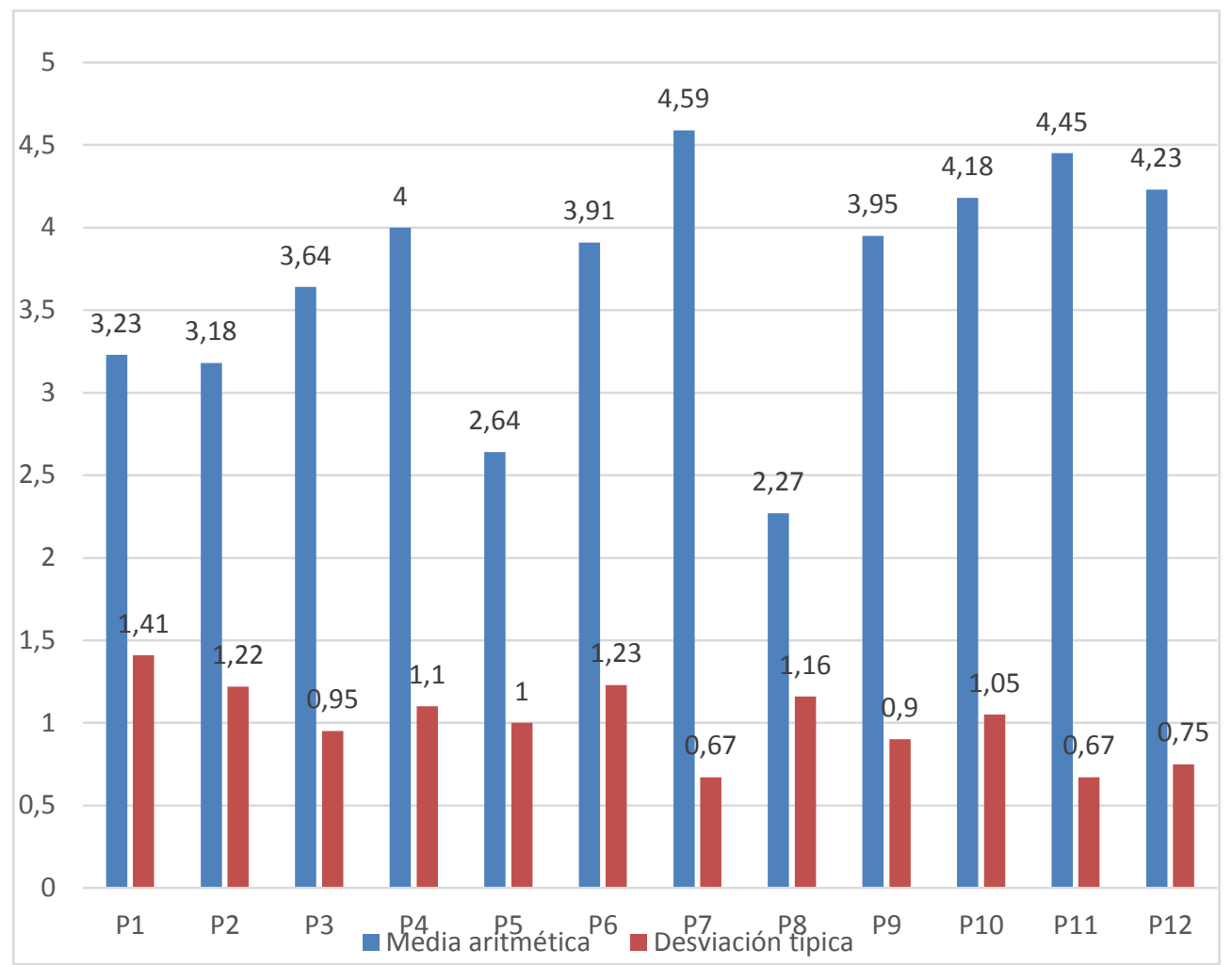

Fig. 5 Medias aritméticas y desviaciones típicas de las preguntas

Si comparamos las puntuaciones en función del sexo del encuestado (ver figura 6) se observa que la tendencia en las puntuaciones es la misma para las personas de ambos sexos, aunque en todas ellas la puntuación del sexo femenino es superior a la del masculino. 


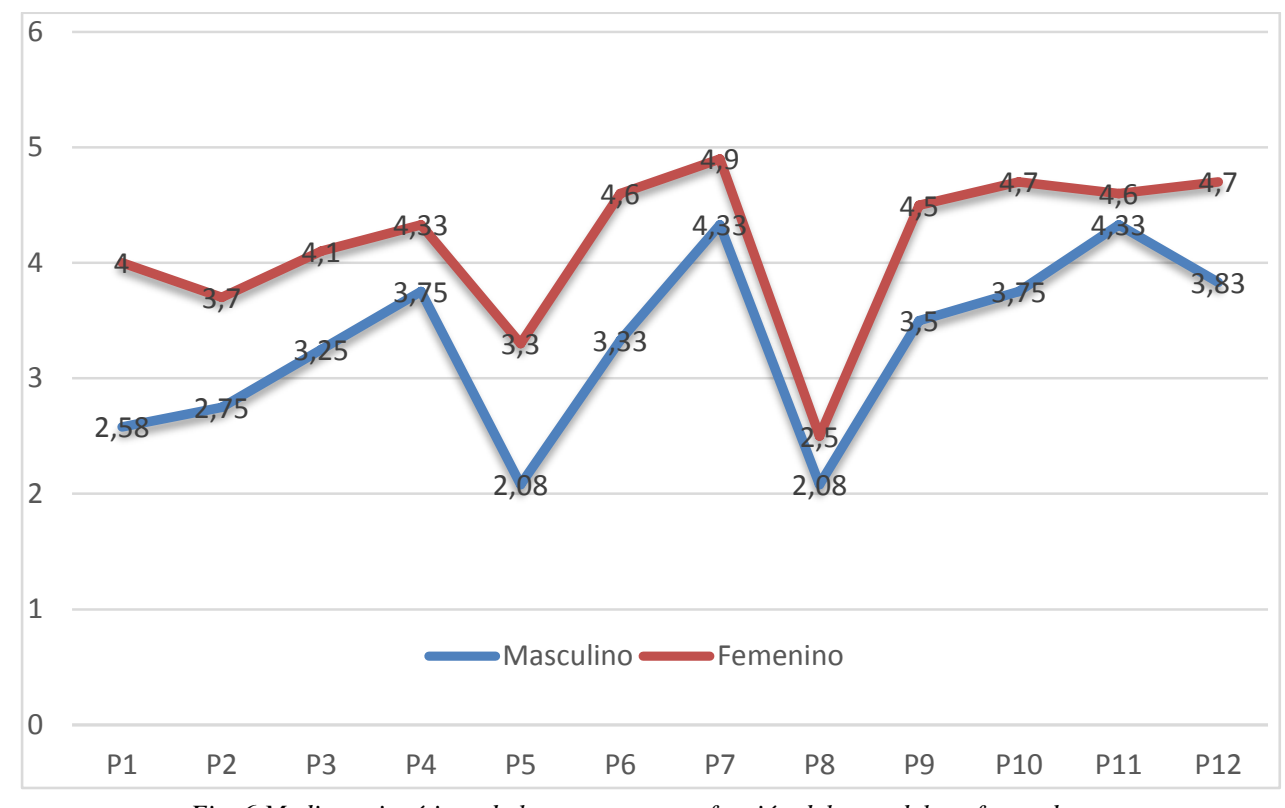

Fig. 6 Medias aritméticas de las preguntas en función del sexo del profesorado

A la vista de los resultados mostrados en el gráfico anterior, para poder indicar una dependencia del sexo con la puntuación, primero se comprobó empíricamente. Se contrastó la normalidad de los datos obtenidos en las 12 preguntas relativas al campus virtual, mediante el test de Saphiro-Wilk, puesto que la muestra tiene menos de 50 registros y este estadístico es el más adecuado. De las 12 variables, únicamente la variable P2 sigue una distribución normal. Por este motivo se consideró la conveniencia de aplicar pruebas no paramétricas en el estudio. En concreto, se estudió la dependencia de las puntuaciones de las 12 respuestas en función del sexo de la persona encuestada, mediante la prueba $U$ de Mann-Whitney-Wilcoxon. La hipótesis nula en este caso, es considerar que para cada una de las 12 preguntas su distribución es la misma independientemente del sexo del encuestado.

La tabla 3 muestra los resultados obtenidos en el p-value para cada una de las 12 preguntas especificadas en la tabla 1.

Tabla 3. Resultados prueba U de Mann-Whitney-Wilcoxon

\begin{tabular}{|l|c|c|c|c|c|c|}
\hline variable & $\mathrm{P} 1$ & $\mathrm{P} 2$ & $\mathrm{P} 3$ & $\mathrm{P} 4$ & $\mathrm{P} 5$ & $\mathrm{P} 6$ \\
\hline$p$-value & $3,503 * 10^{-4}$ & $1,758 * 10^{-4}$ & $4,640^{*} 10^{-5}$ & $6,817 * 10^{-5}$ & $1,340^{*} 10^{-4}$ & $5,161 * 10^{-5}$ \\
\hline variable & $\mathrm{P} 7$ & $\mathrm{P} 8$ & $\mathrm{P} 9$ & $\mathrm{P} 10$ & $\mathrm{P} 11$ & $\mathrm{P} 12$ \\
\hline$p$-value & $2,625 * 10^{-5}$ & $6,562 * 10^{-3}$ & $2,974 * 10^{-5}$ & $2,239 * 10^{-5}$ & $2,676^{*} 10^{-5}$ & $1,784 * 10^{-5}$ \\
\hline
\end{tabular}

Tal como puede observarse, todos los valores del p-value son inferiores a 0,05 , lo cual significa que se rechaza la hipótesis nula de igual distribución en los dos sexos para todas las variables y se comprueba empíricamente esta dependencia, que se aprecia visualmente en la figura 6.

\section{Conclusiones}

Con el objetivo de lograr una mejora de la calidad en la enseñanza y en el aprendizaje, el trabajo desarrollado por Nicolaou et al. (2005) propone diversos escenarios para 
implementar en la universidad. Estos escenarios van desde el apoyo complementario de los procedimientos de enseñanza tradicionales con recursos de e-learning -a través de una plataforma adecuada y mediante medidas de apoyo necesarias para los docentes interesados-, hasta el desarrollo de una estrategia de aprendizaje a distancia, ya sea a partir del desarrollo de nuevos programas de estudio o de módulos mejorados por la tecnología dentro de los programas existentes.

En línea con lo expuesto en el párrafo anterior, desde la UdA se introdujo una nueva metodología de enseñanza y de aprendizaje en el BAE.

Tanto el aprendizaje en línea como el combinado, están destinados a la individualización de la educación (Vanslambrouck, Zhu, Lombaerts, Philipsen y Tondeur, 2018). La atención personalizada podría ser considerada la principal característica de la UdA. Esto resulta relativamente fácil de conseguir, debido a la reducida dimensión de la UdA, que permite tener una relación estrecha con los estudiantes, si bien es cierto que este hecho todavía se ha evidenciado más desde que se incorporó la flexibilidad a partir de las tres modalidades de aprendizaje implementadas en estos estudios.

Tal como se ha expuesto en este trabajo, la media de edad de la primera matrícula realizada a los estudios de BAE es superior a los 28 años y se ha obtenido evidencia empírica de que la modalidad de aprendizaje escogida por los estudiantes está relacionada con la edad.

Este trabajo de investigación también pone de manifiesto que el profesorado considera que el uso del campus virtual del BAE, como herramienta de flexibilización de las metodologías de aprendizaje, rompe las barreras espacio-tiempo, tal como también argumentan Ferro et al. (2009) y Vanslambrouck et al. (2018). Además, según estos últimos autores, se debe buscar el equilibrio entre la enseñanza presencial y la virtual, y el profesorado debe orientar correctamente a los estudiantes y permitirles la flexibilidad necesaria para desarrollar su trabajo.

También se destaca la elevada valoración del acceso rápido a la información y la facilidad para realitzar actividades complementarias de apoyo al aprendizaje, igual que en el estudio de Ferro et al. (2009). Y en línea con estos autores, en este estudio también se pone de manifiesto que la utilización del campus virtual, a pesar de facilitar la realización de actividades complementarias de apoyo al aprendizaje, no permite que el profesorado tenga más tiempo para realitzar otras tareas.

Contrariamente a lo que argumenta la literatura y un ejemplo de ello es el estudio de Ferro et al. (2009), según este trabajo, el profesorado no piensa que el uso del campus virtual aumente el interés y la motivación de los estudiantes.

Los resultados alcanzados en este trabajo presentan algunas limitaciones como consecuencia del reducido tamaño de la UdA y del análisis de tan solo una parte de su profesorado, como es el del BAE. La opinión de un pequeño grupo de docentes universitarios no puede extrapolarse a la totalidad del sistema universitario, ni tan solo a la totalidad de las formaciones que ofrece la UdA con características similares.

En este sentido, como posibles futuras líneas de investigación, por un lado, se podría replicar la investigación en otras universidades de los países vecinos, con el objetivo de identificar si existe una homogeneidad en las opiniones del profesorado, en cuanto a las modalidades de aprendizaje descritas en este estudio. Por otro lado, se podría replicar este trabajo teniendo en cuenta la opinión de los estudiantes, tal como se ha hecho en otros estudios y determinar si los estudiantes tienen la misma opinión que el profesorado, en cuanto a los principales aspectos de estas modalidades de aprendizaje. 


\section{Referencias}

ALABART VILÀ, A., ESCOFET ROIG, A. y VILÀ BOSQUED, G. (2008). Enseñar y aprender con TIC en la Universidad. Barcelona: Colección Cuadernos de Docencia Universitaria ICE-Octaedro.

ALEBAIKAN, R. y TROUDI, S. (2010). "Blended learning in Saudi universities: challenges and perspectives". Research in Learning Technology, vol. 18, issue 1, p. 49-59.

AREA, M. y ADELL, J. (2009). "ELearning: Enseñar y Aprender en Espacios Virtuales” en De Pablos Pons J. (Coord.), La tecnología educativa en el siglo XXI. Málaga: Editorial Aljibe.

AREA, M., SAN NICOLÁS, M. y FARIÑA, B. (2010). "Buenas prácticas de aulas virtuales en la docencia universitaria semipresencial. Teoría de la Educación”. Educación y Cultura en la Sociedad de la Información, vol. 11, issue 3, p. 7-31.

BOPA (2008). Llei 12/2008, del 12 de juny, d'ordenació de l'ensenyament superior. Butlletí Oficial del Principat d'Andorra, núm. 54, any 20, del 16 de juliol. Principat d'Andorra.

BOPA (2010). Decret d'establiment del títol estatal de bàtxelor en administració d'empreses. Butlletí Oficial del Principat d'Andorra, núm. 9, any 22, del 17 de febrer. Principat d'Andorra.

BOPA (2011). Decret d'aprovació del pla d'estudis del bàtxelor en administració d'empreses de la Universitat d'Andorra. Butlletí Oficial del Principat d'Andorra, núm. 25, any 23, del 20 d'abril. Principat d'Andorra.

CASTAÑO, R., JENARO, C. y FLORES, N. (2017). "Percepciones de estudiantes del Grado de Maestro sobre el proceso y resultados de la enseñanza semipresencial: Blended Learning”. Revista de Educación a Distancia, núm. 52, artic. 2.

$<$ http://www.um.es/ead/red/52/castano_et_al.pdf> [Consulta: 18 de enero de 2018].

CRES (2007). Enquesta als joves d'Andorra. Sant Julià de Lòria: Pagés editors.

FERRO, C., MARTÍNEZ, A.I. y OTERO, M.C. (2009). "Ventajas del uso de las TICs en el proceso de enseñanza-aprendizaje desde la óptica de los docentes universitarios españoles". EDUTEC. Revista Electrónica de Tecnología Educativa, número 29. $<$ http://www.edutec.es/revista/index.php/edutec-e/article/view/451/185> [Consulta: 27 de enero de 2016].

FORMA (2007). La formació continuada a Andorra. Model organitzatiu i necessitats formatives. Andorra la Vella: Ecograf.

GARCÍA-VALCÁRCEL， A., HERNÁNDEZ, A. y RECAMAN, A. (2012). "La metodología del aprendizaje colaborativo a través de las TIC: una aproximación a las opiniones de profesores y alumnos”. Revista Complutense de Educación, vol. 23, issue 1, p. 161-188.

GOVERN D'ANDORRA. Departament d'estadística.

$<$ http://www.estadistica.ad/serveiestudis/web/index.asp?lang=4> [Consulta: 24 de marzo de 2018].

LAI, M., LAM, K.M. y LIM, C.P. (2016). "Design principles for the blend in blended learning: a collective case study”. Teaching in Higher Education, vol. 21, issue 6, p. 716729. 
LEVIS, D. (2011). "Redes Educativas 2.1. Medios sociales, entornos colaborativos y procesos de enseñanza y aprendizaje”. Revista de Universidad y Sociedad del Conocimiento, vol. 8, issue 1, p. 7-24. <https://rusc.uoc.edu/rusc/es/index.php/rusc/article/download/v8n1levis/965-1493-1-PB.pdf> [Consulta: 22 de noviembre de 2017].

LÓPEZ-PÉREZ, M.V., PÉREZ-LÓPEZ, M.C. y RODRÍGUEZ-ARIZA, L. (2011). "Blended learning in higher education: Students' perceptions and their relation to outcomes”. Computers \& Education, vol. 56, issue 3, p. 818-826.

MARTÍNEZ-CARO, E. (2011). "Factors affecting effectiveness in e-learning: An analysis in production management courses”. Computer Applications in Engineering Education, vol 19, issue 3, p. 572-581.

MARTÍNEZ-CARO, E., CEGARRA-NAVARRO, J.G. y CEPEDA-CARRIÓN, G. (2015). "An application of the performance evaluation model for e-learning quality in higher education”. Total Quality Management, vol. 26, issue 5-6, p. 632-647.

MAYISELA, T. (2014). “Assessing How E-learning Implementation has Enhanced the Lecturers' Teaching Practices at a South African University”. International Journal of Educational Sciences, vol 6, issue 1, p. 117-124.

MCGORRY, S.Y. (2003). "Measuring quality in online programs”. The Internet and Higher Education, vol. 6, issue 2, p. 159 - 177.

NICOLAOU, C.T., NICOLAIDOU, I.A. y CONSTANTINOU, C.P. (2005). "The eLearning movement as a process of quality improvement in higher education”. Educational Research and Evaluation: An International Journal on Theory and Practice, vol 11, issue 6, p. 605-622.

SINTES, E. (2016). Via Universitària: accés, condicions d'aprenentatge, expectatives i retorns dels estudis universitaris. Barcelona: Fundació Jaume Bofill.

STANDARDS AND GUIDELINES FOR QUALITY ASSURANCE IN THE EUROPEAN HIGHER EDUCATION AREA -ESG- (2015). Brussels, Belgium.

TORRISI-STEELE, G. y DREW, S. (2013). "The literature landscape of blended learning in higher education: the need for better understanding of academic blended practice". International Journal for Academic Development, vol. 18, issue 4, p. 371-383.

TYILO, N. (2017). "E-Learning as Instructional Innovation in Higher Education Institutions (HEI's): Lessons Learnt from the Literature”. Journal of Communication, vol 8, issue 1 , p. 87-93.

VANSLAMBROUCK, S., ZHU, C., LOMBAERTS, K., PHILIPSEN, B. y TONDEUR, J. (2018). "Students' motivation and subjective task value of participating in online and blended learning environments”. The Internet and Higher Education, vol. 36, p. 33-40.

VIGOTSKY, S. (1984). Cientocincuenta Años Después. Infancia y Aprendizaje, (27-28), Madrid: Akal.

WANG, Y.S. (2003). “Assessment of learner satisfaction with asynchronous electronic learning systems”. Information \& Management, vol. 41, issue 1, p. 75-86.

ZUÑIGA, V. y ARNÁEZ SERRANO, E. (2010). “Comunidades virtuales de aprendizaje, espacios dinámicos para enfrentar el Siglo XXI”. Tecnología en Marcha, vol 23, issue 1, p. 19-28. 